ISSN 1997-5902

\title{
Caractéristiques écologiques du peuplement ligneux de la réserve de biosphère du Ferlo (Nord Sénégal)
}

\author{
Daouda NGOM ${ }^{1,2}$, Thioro FALL ${ }^{1}$, Omar SARR ${ }^{2}$, Sékouna DIATTA ${ }^{3}$ et Léonard E. AKPO ${ }^{3}$ \\ ${ }^{1}$ Laboratoire d'Agroforesterie et d'Écologie (LAFE) - Département d'Agroforesterie / UFR ST / Université Assane \\ Seck de Ziguinchor (Sénégal) \\ ${ }^{2}$ Laboratoire d'Écologie et d'Ecohydrologie / Département de Biologie végétale / FST / Université Cheikh Anta Diop \\ de Dakar \\ ${ }^{1}$ Correspondant : Tél : 00 (221) 77657 15 37, courriel : ngom_daouda@yahoo.fr
}

Original submitted in on $16^{\text {th }}$ May 2013 Published online at www.m.elewa.org on $29^{\text {th }}$ May 2013.

http://dx.doi.org/10.4314/jab.v65i0.89644

\section{RESUME :}

Objectifs : Cette étude a pour objectif la caractérisation des différents paramètres structuraux du peuplement ligneux de la réserve de biosphère du Ferlo (RBF).

Méthodes et résultats : En 2011, nous avons procédé à une mesure des caractéristiques dendrométriques des arbres et un inventaire de la flore ligneuse sur 110 placettes de $900 \mathrm{~m}^{2}$ chacune.

La richesse spécifique totale est de 49 espèces et la richesse spécifique moyenne est de 5,8 espèces/relevé. L'analyse des fréquences centésimales montre que Guiera senegalensis (75\%), Combretum glutinosum $(65,5 \%)$, Boscia senegalensis $(63,6 \%)$ et Pterocarpus lucens $(60,9 \%)$ sont les espèces les plus fréquentes. La densité d'arbres est plus élevée dans l'aire centrale (392 ind/ha) alors que la surface terrière et le recouvrement sont plus élevé dans la zone tampon avec respectivement $9,17 \mathrm{~m}^{2}$.ha-1 et $43 \%$. Du point de vue de la structure, le peuplement ligneux comporte une forte proportion d'individus relativement jeunes. En effet, plus de $90 \%$ des individus inventoriés ont des hauteurs comprises entre 0 et $6 \mathrm{~m}$ et de circonférence comprises entre 10 et 100 $\mathrm{cm}$, ce qui montre l'importance de la strate arbustive dans la RBF. Du point de vue de l'importance écologique, trois espèces se distinguent : Pterocarpus lucens (18\%), Guiera senegalensis (16\%) et Combretum glutinosum (13\%). Le taux de régénération du peuplement végétal est de $72 \%$ dans l'ensemble de la RBF. II est deux fois plus élevé dans l'aire centrale (79\%), moins anthropisée, que dans la zone tampon (36\%) et l'aire de transition (39\%). Guiera senegalensis présente le potentiel de régénération le plus élevé avec un indice spécifique de régénération de plus de $62 \%$.

Conclusion et applicabilité des résultats : Ces résultats témoignent des potentialités de la RBF à assurer le double rôle de conservation de la biodiversité et de fourniture de services écosystèmiques aux communautés locales.

Mots clés : cortège floristique, peuplement ligneux, importance écologique, densité, recouvrement

\section{ABSTRACT}

Objectives : This study aims to characterize the various structural parameters of the woody vegetation in Ferlo Biosphere Reserve (FBR).

Methods and Results: In 2011 measurement of dendrometric characteristics of trees and an inventory of the woody flora was carried out in 110 plots of $900 \mathrm{~m}^{2}$ each. The total specific richness was 49 species and the 
average of the specific richness was 5.8 species / plot. The analysis of centesimal frequencies showed that Guiera senegalensis (75\%), Combretum glutinosum (65.5\%), Boscia senegalensis (63.6\%) and Pterocarpus lucens $(60.9 \%)$ are the most common species. Tree density is higher in the central area (392 ind / ha) while the basal area and the recovery are higher in the buffer zone with respectively $9.17 \mathrm{~m}^{2} /$ ha and $43 \%$. Concerning the structure, the woody trees have a relatively high proportion of seedlings. In fact, over $90 \%$ of trees surveyed have heights between 0 and $6 \mathrm{~m}$ and circumference between 10 and $100 \mathrm{~cm}$, which shows the importance of the shrub layer in the FBR. With regard to the ecological importance, three species are distinguished: Pterocarpus lucens (18\%), Guiera senegalensis (16\%) and Combretum glutinosum (13\%). The rate of regeneration of plant population is $72 \%$ in the FBR. It is twice higher in the core area (79\%) which is less anthropized than the buffer zone (36\%) and the transition zone (39\%). Guiera senegalensis has the highest potential of regeneration with a specific regeneration index more than $62 \%$.

Conclusion and applicability of the results: These results demonstrate the potential of the FBR to ensure the dual role of biodiversity conservation and provision of ecosystem services to local communities.

Key words : floristic cortege, wood stand, ecological importance, density, recovery

\section{INTRODUCTION}

Dans les écosystèmes sylvopastoraux du Ferlo, les ressources végétales jouent un rôle essentiel dans l'économie rurale; elles contribuent à l'apport de protéines, de minéraux et de vitamines indispensables à l'équilibre alimentaire des hommes et des animaux, à fournir divers autres services (énergie domestique, bois de service, plantes médicinales). Elles contribuent aussi, à l'accroissement de la productivité des terres et au maintien de l'équilibre des écosystèmes (Ngom, 2013). Cependant, ces milieux subissent depuis plusieurs décennies de fortes perturbations liées d'une part aux conditions naturelles d'aridité, longue saison sèche, forte évaporation, forte variabilité spatio-temporelle des faibles précipitations (Toupet,

\section{MATERIEL ET MÉTHODES}

La zone d'étude : L'étude a été menée dans la réserve de biosphère du Ferlo dans la partie septentrionale du Sénégal entre $14^{\circ} 24^{\prime}-16^{\circ} 11^{\prime}$ de latitude $\mathrm{N}$ et $13^{\circ} 07^{\prime}$ $14^{\circ} 51^{\prime}$ de longitude $W$ (figure 1). Les reserves de biosphère sont des aires portant sur des écosystèmes terrestres et côtiers/marins qui visent à promouvoir des solutions pour réconcilier la conservation de la biodiversité avec son utilisation durable (UNESCO, 1996). La réserve de biosphère du Ferlo joue principalement trois fonctions (conservation de la
1989 ; Le Houérou, 1989) et d'autre part à une surexploitation non contrôlée des ressources ( Grouzis \& Albergel, 1991), qui accentuent la péjoration des conditions climatiques. La connaissance des caractéristiques du peuplement végétal permet de mieux appréhender les écosystèmes sylvopastoraux, de les décrire dans leurs aspects les plus divers afin de proposer des stratégies de gestion durable. La présente étude se propose d'analyser la variabilité structurale du peuplement ligneux et de caractériser la végétation ligneuse en évaluant la densité, le couvert ligneux et l'importance écologique des espèces dans les différentes zones de la réserve de biosphère du Ferlo.

biodiversité, développement économique et humain durable et production de savoirs) et est structurée selon trois zones interconnectées à savoir l'aire centrale, la zone tampon et l'aire de transition. Le Ferlo correspond aux formations sableuses dunaires du continental terminal. Le relief, peu accentué avec des pentes inférieures à 3\% (Cornet 1992) joue un rôle dans l'évolution des sols. II permet de distinguer trois principaux types de sols: Les sols sableux, les sols argilo-sableux à argileux et les sols latéritiques cuirassés. 


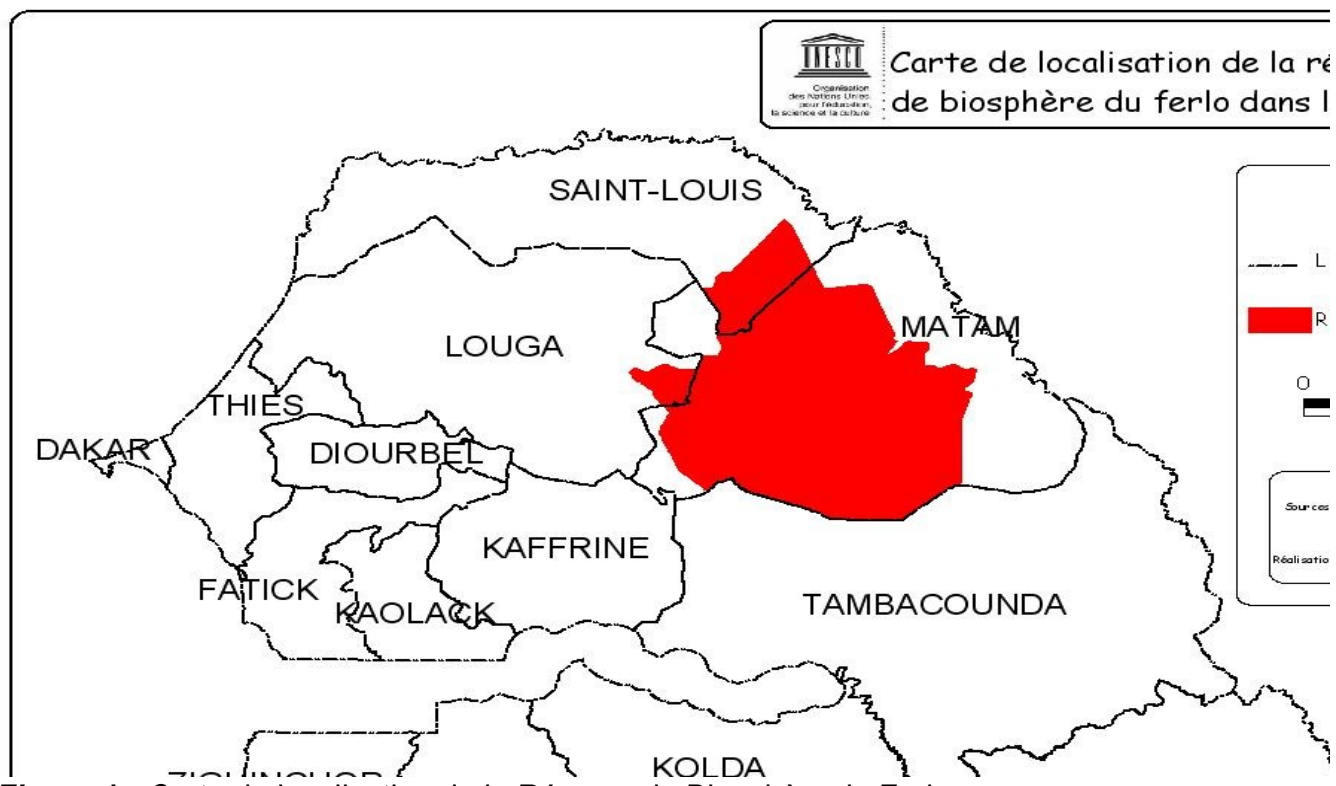

Figure 1 : Carte de localisation de la Réserve de Biosphère du Ferlo

Le climat est tropical sec, de type soudano-sahélien, caractérisé par des pluies qui s'étendent de juin à octobre, permettant de distinguer classiquement dans l'année deux périodes : une saison sèche $(P<0,35 E T P)$ de 7 à 9 mois (octobre à mai) et une saison des pluies $(P \geq 0,35 \mathrm{ETP})$ de 3 à 5 mois (Ngom, 2008). Les données pluviométriques de la station de référence de Ranerou montrent que la moyenne des précipitations sur 51 années (1960-2011) est de 474 mm/an pour 29 jours de pluie en moyenne (Fig. 2). L'année la plus humide de la série observée, 1969, a enregistré $845 \mathrm{~mm}$ pour 36 jours de pluie, en revanche les deux années les plus déficitaires sont $1983(254 \mathrm{~mm})$ et 1971 (275 mm). Ces deux années correspondent aux deux grandes sécheresses du Sahel. La température moyenne annuelle s'établit à $28,6^{\circ}$ mais avec des amplitudes thermiques élevées. Les valeurs moyennes des températures minimales et maximales mensuelles sont respectivement de $17^{\circ} \mathrm{C}$ (décembre) et $43^{\circ} \mathrm{C}$ (mai).

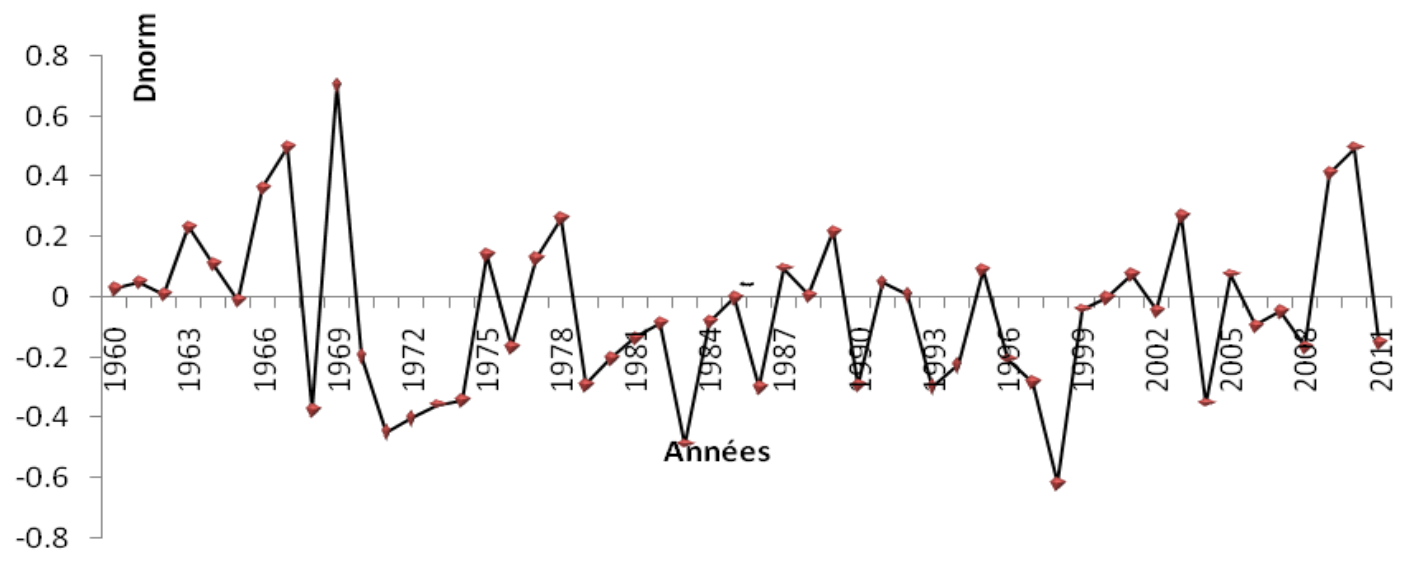

Figure 2: Analyse des tendances évolutives de la pluviosité annuelle par la méthode de la différence normalisée 
Relevés de végétation: La carte de zonage de la réserve de biosphère a été la base d'échantillonnage. L'échantillonnage a utilisé la méthode des transects. Au total sept transects d'orientation W-E de longueurs différentes et distant de $4 \mathrm{~km}$ ont été choisis (figure 3).

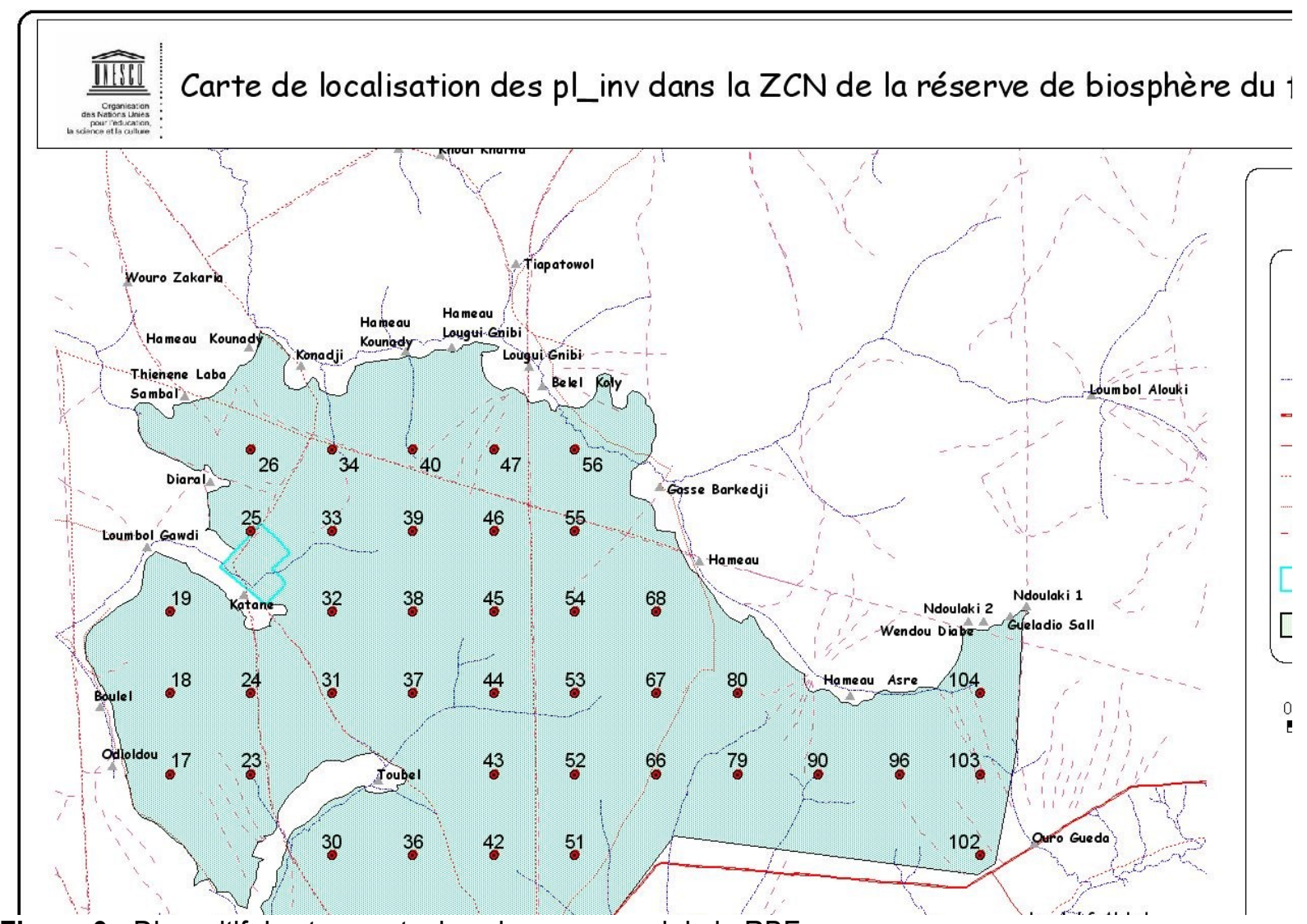

Figure 3 : Dispositif des transects dans le noyau nord de la RBF

Dans l'ensemble de la RBF, 110 relevés ont été échantillonnés dont 57 relevés dans l'aire centrale, 28 en zone tampon et 25 dans l'aire de transition. L'unité d'échantillonnage est une placette carrée de $30 \mathrm{~m} \times 30 \mathrm{~m}$ soit une aire de relevée de $900 \mathrm{~m}^{2}$ (Boudet, 1984) pour l'étude de la végétation sahélo-soudanienne.

Dans chaque relevé, un recensement exhaustif des ligneux a été effectué. Des mensurations dendrométriques ont été réalisées pour évaluer quelques paramètres dimensionnels :

- La circonférence à la base du tronc à $30 \mathrm{~cm}$ du sol du fait que beaucoup d'arbres du Ferlo sont multicaules et présentent des ramifications.

- Le diamètre de la projection du houppier au sol dans deux directions (nord-sud et est-ouest)

- La hauteur des arbres

- La distance entre deux arbres par la méthode du plus proche individu
Les éléments topographiques, le sol et le substrat géologique du peuplement sont également relatés.

Les échantillons botaniques ont été identifiés sur le terrain ou en laboratoire à l'aide de la "Flore du Sénégal" (Berhaut, 1967) et de l'ouvrage Arbres, arbustes et lianes d'Afrique de l'Ouest (Arbonnier, 2002). Les synonymes ont été actualisés et normalisés sur la base de l'énumération des plantes à fleurs d'Afrique Tropicale (Lebrun \& Stork, 1991, 1992, 1995, 1997).

Traitement des données :

Paramètres de la végétation ligneuse : Les données obtenues à partir des relevés de végétation ont été traitées à l'aide du tableur Excel et du logiciel XLstat qui ont servi au classement des données numériques et à l'élaboration des graphiques. Ils ont été aussi utilisés pour calculer les paramètres de caractérisation de la végétation que sont la densité relative, la surface terrière, le recouvrement, la dominance relative, la fréquence 
relative et l'importance écologique dans les différentes zones de la réserve de biosphère. Les formules ci-après ont été utilisées pour procéder au calcul de ces paramètres :

- $\quad$ La richesse spécifique a été évaluée à partir de la richesse spécifique totale et la richesse spécifique moyenne. La richesse spécifique totale (S) est le nombre total d'espèces que comporte le peuplement considéré dans un écosystème donné (Ramade, 2003). La richesse spécifique moyenne correspond au nombre moyen d'espèces par relevé pour un échantillon donné.

- L'Analyse fréquentielle: L'Analyse fréquentielle est une méthode qui consiste à apprécier la distribution des espèces à travers les relevés. La fréquence de présence renseigne sur la distribution d'une espèce dans un peuplement. Elle peut être exprimée en valeur absolue ou en pourcentage (\%). En \%, elle est estimée par la formule suivante (Roberts-Pichette et Gillespie, 2002 cité par Gning, 2008) :

$\mathrm{F}=\frac{\mathrm{Nri}}{\mathrm{Nr}} \times 100$

$\mathrm{F}=$ fréquence de présence exprimée en pourcentage $(\%) ; N_{\mathrm{ri}}=$ nombre de relevés où l'on retrouve l'espèce i et $\mathrm{N}_{\mathrm{r}}=$ nombre total de relevés.

- $\quad$ La densité : est le nombre d'individus par unité de surface. Elle s'exprime en nombre d'individus/ha. Nous avons déterminé la densité observée et la densité théorique.

- $\quad$ La densité observée ou densité réelle est obtenu par le rapport de l'effectif total des individus dans l'échantillon (N) par la surface échantillonnée (S). Dob. $=\frac{\mathrm{N}}{\mathrm{s}}$

- $\quad$ La densité théorique (Dth) est obtenue à partir de la distance moyenne $(\mathrm{dm})$ entre les arbres. Elle ne tient pas compte d'éventuelles irrégularités sur la parcelle, et de présence de zones sans arbres. Dth. $=\left(\frac{100}{\mathrm{dm}}\right)^{2}$ $d m=$ distance moyenne entre les arbres ; $(100)^{2}$ correspond à $10000 \mathrm{~m}^{2}$ soit 1 ha.

- Le couvert ligneux est la surface de la couronne de l'arbre projetée verticalement au sol. II est exprimé en mètre carré par hectare $\left(\mathrm{m}^{2} \cdot\right.$ ha. $\left.{ }^{-1}\right)$. Le couvert ligneux est calculé avec la formule ci-dessous :

$\mathrm{C}=\frac{\sum \pi\left(\frac{\mathrm{d} \mathrm{mh}}{2}\right)^{2}}{\mathrm{~S}_{\mathrm{E}}} \begin{aligned} & \text { Avec } C=\text { couvert ligneux } ; d_{m b}=\text { diamètre moyen du houppier en } \mathrm{m} ; S=\text { surface } \\ & \text { de l'échantillon considéré en ha. }\end{aligned}$

- $\quad$ La surface terrière désigne la surface de l'arbre évaluée à la base du tronc de l'arbre. Elle est exprimée en mètre carré par hectare $\left(\mathrm{m}^{2}\right.$.ha. $\left.{ }^{-1}\right)$. Elle est donc obtenue à partir de la formule suivante :

$\mathrm{St}_{\mathrm{t}}=\frac{\sum \pi\left(\frac{\mathrm{d} 0,3}{2}\right)^{2}}{\mathrm{SE}_{\mathrm{E}}} \quad \begin{aligned} & \text { Avec } S_{t}=\text { surface terrière ; do,3 }=\text { diamètre en } \mathrm{m} \text { du tronc à } 0,3 \mathrm{~m} ; S_{E}= \\ & \text { surface de l'échantillon considéré en ha. }\end{aligned}$

- L'Indice de Valeur d'Importance des espèces (IVI) a été mis au point par Curtis et Macintosh (1950) comme étant la somme de la fréquence relative, la densité relative et la dominance relative. II est une expression synthétique et quantifiée de l'importance d'une espèce dans un peuplement.

Pour une interprétation plus facile de l'IVI, Lindsey (1956) cité par Labat (1995) l'a exprimé en pourcentage (\%) en le définissant comme la moyenne arithmétique, pour l'espèce i, de la densité relative (Dr), la fréquence relative (Fr) et la dominance relative (Domr).

$$
\mathrm{IVI}=\frac{\mathrm{Dr}+\mathrm{Fr}+\mathrm{Domr}}{3}
$$

- Le taux de régénération du peuplement est donné par le rapport en pourcentage entre l'effectif total des jeunes plants (circonférence $<10 \mathrm{~cm}$ ) et l'effectif total du peuplement (Poupon, 1980) : 
$\mathrm{TRP}=\frac{\text { Effectif total des jeunes plants }}{\text { Effectif total du peuplement }} \times 100$

L"effectif total du peuplement regroupant aussi bien les jeunes plants que les plantes adultes.

\section{- $\quad$ L'Importance spécifique de régénération est} quant à elle obtenue à partir du rapport en pourcentage entre l'effectif des jeunes plants d'une espèce et l'effectif total des jeunes plants dénombrés (Akpo \& Grouzis, 1996) :
ISR $=$

Effectif des jeunes plants d'une espèce Effectif total des jeunes plants dénombrés

Pour étudier le potentiel de régénération naturelle du peuplement ligneux dans les différentes zones de la RBF, nous avons considéré tous les sujets dont la circonférence est inférieure ou égale à $10 \mathrm{~cm}$ comme appartenant à la régénération (Akpo \& Grouzis, 1996).

\section{RESULTATS}

La richesse spécifique : La richesse spécifique représente une des principales caractéristiques d'un peuplement végétal, et représente la mesure la plus fréquemment utilisée pour étudier la biodiversité. La richesse spécifique totale (nombre totale d'espèces) à l'échelle de la RBF est de 49 espèces. Elle varie de 35 espèces dans l'aire centrale et l'aire de transition à 28 espèces en zone tampon (tableau 1).

Tableau 1 : Variation de la richesse spécifique dans les différentes zones de la RBF

\begin{tabular}{|l|c|c|c|c|}
\cline { 2 - 5 } \multicolumn{1}{c|}{} & Aire centrale & Zone tampon & Aire de transition & RBF \\
\hline Richesse spéc. totale (S) & 35 & 28 & 35 & $\mathbf{4 9}$ \\
\hline Richesse spéc. Moyenne (Ś) & 5,4 & 6 & 6,6 & $\mathbf{5 , 8}$ \\
\hline Variance de la richesse spéc. moy. & 4,03 & 8,73 & 10,58 & $\mathbf{7 , 1 1}$ \\
\hline
\end{tabular}

La richesse spécifique moyenne est de 5,8 espèces /relevé à l'échelle de la réserve de biosphère. Elle a également variée en fonction des différentes zones. Elle est en moyenne plus élevée dans l'aire de transition $(6,6$ espèces/relevé) que dans la zone tampon (6 espèces/relevé) et l'aire centrale (5,4 espèce/relevé). La variance de la richesse spécifique moyenne est importante pour déterminer l'homogénéité du peuplement. Elle est deux fois plus élevée dans l'aire de transition $(10,58)$ et la zone de tampon $(8,73)$ que dans l'aire centrale $(4,03)$. Plus la variance de la richesse spécifique moyenne est élevée, plus l'hétérogénéité du peuplement est forte.

Les paramètres structuraux de la végétation

L'analyse fréquentielle: La flore d'un milieu est l'énumération et la description de toutes les espèces qui y croissent. Dans la RBF, la flore est riche de 49 espèces avec des fréquences centésimales variables dans les différentes zones (tableau 2). L'examen du tableau montre que dans la RBF aucune espèce n'est présente dans tous les relevés, c'est dire une fréquence de 100\%. Une seule espèce (Guiera senegalensis) est présente dans les $3 / 4$ des relevés. Elle est suivie de Combretum glutinosum $(65,5 \%)$, Boscia senegalensis $(63,6 \%)$ et Pterocarpus lucens $(60,9 \%)$. Cette répartition des espèces est variable en fonction des différentes zones. Dans l'aire centrale Boscia senegalensis qui est présente dans plus de huit relevés sur dix est la deuxième espèce la plus fréquente derrière Guiera senegalensis (86\%). Dans la zone tampon Combretum glutinosum (84\%) devient l'espèce la plus fréquente et est suivie par Guiera senegalensis (68\%). Dans l'aire de transition Combretum glutinosum présente la fréquence la plus élevée de toutes les zones avec $89,3 \%$. Les espèces qui présentent les fréquences les plus faibles sont celles qui sont exclusives à une des trois zones de la réserve de biosphère.

Tableau 2 : Fréquences centésimales des espèces ligneuses dans les différentes zones de la RBF 
Ngom et al J. Appl. Biosci. 2013. Caractéristiques écologiques du peuplement ligneux de la réserve de biosphère du Ferlo, Sénégal

\begin{tabular}{|c|c|c|c|c|}
\hline Zones & Aire centrale & Zone tampon & Aire de transition & RBF \\
\hline Guiera senegalensis J. F. Gmel. & 86,0 & 68 & 60,7 & 75,5 \\
\hline Combretum glutinosum Perrott. ex DC. & 45,6 & 84 & 89,3 & 65,5 \\
\hline Boscia senegalensis (Pers.) Lam. Ex Poir. & 82,5 & 32 & 53,6 & 63,6 \\
\hline Pterocarpus lucens Lepr. Ex Guill. et Perrott. & 78,9 & 56 & 28,6 & 60,9 \\
\hline Grewia bicolor Juss. & 63,2 & 48 & 28,6 & 50,9 \\
\hline Combretum micranthum G. Don & 29,8 & 32 & 21,4 & 28,2 \\
\hline Balanites aegyptiaca (L.) Del. & 12,3 & 40 & 46,4 & 27,3 \\
\hline Commiphora africana (A. Rich.) Engl. & 12,3 & 40 & 42,9 & 26,4 \\
\hline Adenium obesum (Forsk.) Roem. et Schult. & 10,5 & 32 & 39,3 & 22,7 \\
\hline Acacia macrostachia Reichenb ex. Benth. & 8,8 & 32 & 25,0 & 18,2 \\
\hline Acacia senegal (L.) Willd & 15,8 & 20 & 21,4 & 18,2 \\
\hline Feretia opodanthera Del. & 15,8 & 16 & 17,9 & 16,4 \\
\hline Boscia angustifolia A. Rich. & 8,8 & 16 & 3,6 & 9,1 \\
\hline Lannea acida A. Rich. & 3,5 & 8 & 21,4 & 9,1 \\
\hline Leptadaena hastata (Pers.) Dcne & 3,5 & 16 & 10,7 & 8,2 \\
\hline Sterculia setigera Del. & 3,5 & 4 & 17,9 & 7,3 \\
\hline Acacia seyal Del. & 8,8 & 0 & 7,1 & 6,4 \\
\hline Dalbergia melanoxylon Guill. et Perrott. & 7,0 & 0 & 7,1 & 5,5 \\
\hline Pterocarpus erinaceus Poir. & 1,8 & 4 & 14,3 & 5,5 \\
\hline Acacia ataxacanta DC. & 5,3 & 4 & 3,6 & 4,5 \\
\hline Adansonia digitata $\mathrm{L}$. & 3,5 & 4 & 7,1 & 4,5 \\
\hline Combretum nigricans Lepr. ex Guoll. et Perrott & 8,8 & 0 & 0 & 4,5 \\
\hline Terminalia avicennoides Guill. \& Perr. & 0 & 12 & 7,1 & 4,5 \\
\hline Acacia laeta R. Br. ex Benth. & 0 & 4 & 10,7 & 3,6 \\
\hline Maytenus senegalensis (Lam.) Exell. & 3,5 & 0 & 7,1 & 3,6 \\
\hline Acacia pennata (L.) Willd. & 0 & 4 & 7,1 & 2,7 \\
\hline Anogeissus leiocarpus (DC) Guill. et Perrott. & 1,8 & 0 & 7,1 & 2,7 \\
\hline Cadaba farinosa Forsk. & 0 & 0 & 10,7 & 2,7 \\
\hline Crataeva adansoni DC. & 0 & 0 & 10,7 & 2,7 \\
\hline Acacia nilotica (L.) Willd. ex Del. & 1,8 & 4 & 0 & 1,8 \\
\hline Asparagus pauli-gulielmi Solms-Laub. & 0 & 0 & 7,1 & 1,8 \\
\hline Calotropis procera (Ait.) Ait. F. & 1,8 & 0 & 3,6 & 1,8 \\
\hline Dichrostachys cinerea L. Wight \& Arn. & 0 & 4 & 3,6 & 1,8 \\
\hline Gardenia erubescens Stapf. & 3,5 & 0 & 0 & 1,8 \\
\hline Grewia flavescens Juss. & 1,8 & 0 & 0 & 1,8 \\
\hline Sclerocarya birrea (A. Rich.) Hochst. & 1,8 & 0 & 3,6 & 1,8 \\
\hline Ziziphus mauritiana Lam. & 3,5 & 0 & 0 & 1,8 \\
\hline Ziziphus mucronata Willd. & 0 & 8 & 0 & 1,8 \\
\hline Acacia ehrenbergiana Hayne & 1,8 & 0 & 0 & 0,9 \\
\hline Acacia raddiana Savi & 0 & 0 & 3,6 & 0,9 \\
\hline Piliostigma reticulatum (DC.) Hochst. & 1,8 & 0 & 0 & 0,9 \\
\hline Bombax costatum Pellegr. et Vuillet & 1,8 & 0 & 0 & 0,9 \\
\hline Combretum aculeatum Vent. & 0 & 0 & 3,6 & 0,9 \\
\hline Entada africana Guill. et Perrott. & 0 & 4 & 0 & 0,9 \\
\hline
\end{tabular}




\begin{tabular}{|l|l|l|l|l|}
\hline Gardenia tern folia Schumach et Thonn. & 0 & 0 & 3,6 & 0,9 \\
\hline Maerua angolensis DC. & 0 & 4 & 0 & 0,9 \\
\hline Maerua crassifolia Forsk. & 1,8 & 0 & 0 & 0,9 \\
\hline Mitragyna inermis (Willd.) O. Ktze. & 1,8 & 0 & 0 & 0,9 \\
\hline Strychnos spinosa Lam. & 0 & 4 & 0 & 0,9 \\
\hline
\end{tabular}

La densité : La densité observée (densité réelle) et la densité théorique des différentes zones de la réserve de biosphère sont consignées dans le tableau 3 . Dans la RBF la densité observée est de 389 pieds/hectare. Cependant, elle est plus élevée dans l'aire centrale (392) que dans la zone tampon (352) et l'aire de transition (347). Les espèces qui présentent les plus fortes densités ont varié d'une zone à l'autre.

- Dans l'aire centrale: Guiera senegalensis, Pterocarpus lucens et Boscia senegalensis avec respectivement des densités de $154 ; 63$ et 50 individus par hectare. Deux espèces (Guiera senegalensis et Pterocarpus lucens) représentent plus de la moitié de la densité moyenne de l'aire centrale (214 pieds / ha).

- Dans la zone tampon: Guiera senegalensis, Balanites aegyptiaca et Combretum glutinosum avec respectivement 76 pieds / ha, 47 pieds / ha et 45 pieds / ha.

- Dans l'aire de transition: Combretum glutinosum, Guiera senegalensis, Boscia senegalensis, avec respectivement 113 pieds / ha, 48 pieds / ha et 39 pieds / ha sont les espèces les plus présentes dans cette zone. La densité théorique est plus élevée que la densité observée dans les différentes unités de la réserve de biosphère. Le rapport entre la densité théorique et la densité observée est de 2,22 dans l'ensemble de la réserve de biosphère, mais est variable d'une unité à l'autre. II est plus élevé dans l'aire centrale $(2,48)$ et la zone de transition $(2,57)$ que dans l'aire de transition $(2,1)$ qui subit plus l'influence de l'homme, qui par son action impacte sur l'espacement entre les arbres.

Tableau 3 : Paramètres structuraux de la végétation ligneuse de la RBF

\begin{tabular}{|l|c|c|c|c|}
\hline Paramètres écologiques & Aire centrale & Zone tampon & Aire de transition & RBF \\
\hline Densité observée Dob $\left(\mathrm{n} \cdot \mathrm{ha}^{-1}\right)$ & 392 & 352 & 347 & 389 \\
\hline Dm entre arbres $(\mathrm{m})$ & 3,2 & 3,32 & 3,7 & 3,4 \\
\hline Densité théorique $\left(\mathrm{n} \cdot \mathrm{ha}^{-1}\right)$ & 976 & 907 & 730 & 865 \\
\hline Rapport Dth/Dob & 2,48 & 2,57 & 2,1 & 2.22 \\
\hline Taux de couverture \% & $32 \%$ & $43 \%$ & $35 \%$ & $35,30 \%$ \\
\hline Surface terrière $\left(\mathrm{m}^{2} \cdot \mathrm{ha}^{-1}\right)$ & 6,1 & 9,17 & 8,64 & 7,44 \\
\hline Taux de régénération $\%$ & $79 \%$ & $36 \%$ & $39 \%$ & $72 \%$ \\
\hline
\end{tabular}

Le recouvrement : Le taux de recouvrement est de 35,3 $\%$ dans l'ensemble de la RBF. II varie en fonction des différentes zones car sa valeur dépend fortement de la présence de grands arbres aux larges houppiers. Il est de $32 \%$ pour l'aire centrale, $43 \%$ en zone tampon et $35 \%$ dans l'aire de transition (tableau 3).

Le taux de recouvrement des espèces dominantes dans les différentes zones se présente comme suit:

- $\quad$ Dans l'aire centrale: Pterocarpus lucens (12,5 $\%)$, Guiera senegalensis (6\%), Combretum glutinosum $(2,9 \%)$ et Grewia bicolor $(1,7 \%)$.

- $\quad$ En zone de transition : Pterocarpus lucens (13,7 $\%)$, Guiera senegalensis (5,9\%), Combretum glutinosum $(5,8 \%)$ Acacia senegal (4\%) et Balanites senegalensis $(3,45 \%)$.
- $\quad$ Dans l'aire de transition : Combretum glutinosum (10,14\%) Pterocarpus lucens (3,3\%), Sterculia setigera (3,1\%), Guiera senegalensis (3,1\%) et Acacia senegal $(2,8 \%)$.

La surface terrière : La surface terrière moyenne de la réserve de biosphère est de $7,44 \mathrm{~m}^{2} \cdot$ ha. ${ }^{-1}$. Elle varie de $6,1 \mathrm{~m}^{2} / \mathrm{ha}$ dans l'aire centrale à $9,17 \mathrm{~m}^{2} /$ ha en zone tampon et $8,64 \mathrm{~m}^{2} /$ ha dans l'aire de transition (tableau 3). La surface terrière des espèces dominantes dans les différentes zones se présente comme suit :

- $\quad$ Dans l'aire centrale Pterocarpus lucens (2,33 $\mathrm{m}^{2}$.ha.- $\left.{ }^{-1}\right)$, Adansonia digitata $\left(1,25 \mathrm{~m}^{2}\right.$.ha.-1), Guiera senegalensis $\left(0,45 \mathrm{~m}^{2} / \mathrm{ha}\right)$ présentent les surfaces terrières les plus élevées et contribuent pour prés de $67 \%$ à la surface terrière de la réserve de biosphère. 
- $\quad$ En zone tampon Pterocarpus lucens $\left(4,3 \mathrm{~m}^{2}\right.$.ha.

1), Combretum glutinosum $\left(1,03 \mathrm{~m}^{2}\right.$.ha.- $\left.{ }^{-1}\right)$ et Balanites aegyptiaca $\left(0,62 \mathrm{~m}^{2}\right.$.ha.- $\left.{ }^{-1}\right)$ contribuent pour $65 \%$ à la surface terrière.

- Dans l'aire de transition: Combretum glutinosum $\left(2,1 \mathrm{~m}^{2} / \mathrm{ha}\right)$, Sterculia setigera $\left(1,47 \mathrm{~m}^{2}\right.$. ha. $\left.{ }^{-1}\right)$, Adansonia digitata $\left(\mathrm{m}^{2}\right.$.ha. $\left.{ }^{-1}\right)$ dominent largement car elles représentent plus de la moitié de la surface terrière de la zone de transition.
La structure du peuplement: La structure du peuplement ligneux a été établie par la distribution des ligneux en classe de hauteur et en classe de circonférence dans les différentes zones et globalement dans la réserve de biosphère du Ferlo.

* Distribution selon la hauteur : La distribution des arbres dans la RBF selon les classes de hauteur (figure 4) est de type unimodal et est caractérisée par la présence de toutes les classes de hauteur.

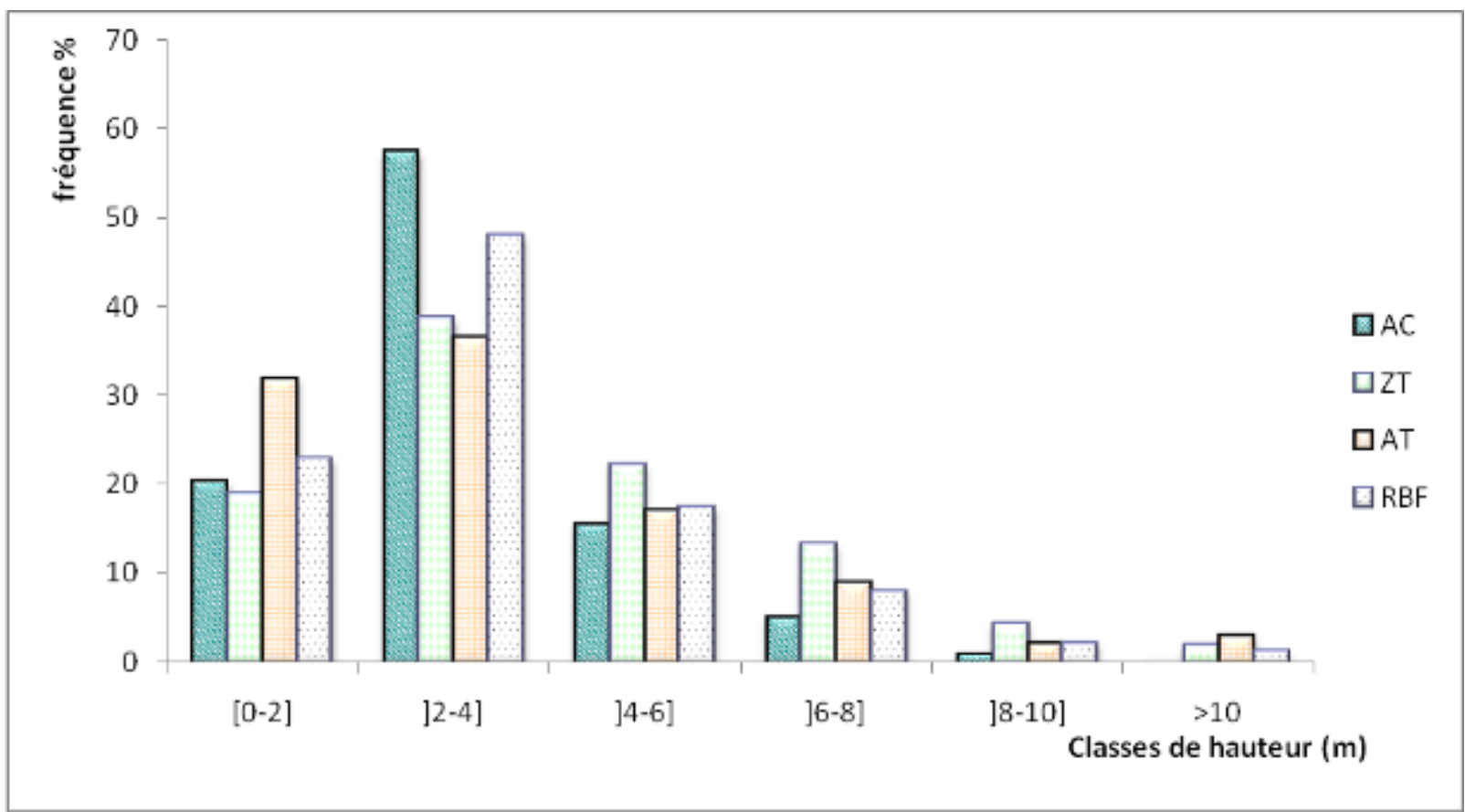

Figure 4 : Distribution des ligneux de la RBF selon les classes de hauteur en $\mathrm{m}$.

$\mathrm{AC}=$ Aire centrale, $\mathrm{ZT}=\mathrm{Z}$ one tampon, $\mathrm{AT}=$ Aire de transition et $\mathrm{RBF}=$ réserve de biosphère du Ferlo

La hauteur des ligneux est comprise entre $0,36 \mathrm{~m}$ et $16,25 \mathrm{~m}$. La classe] 2-4 m] (48\% des ligneux) est la mieux représentée aussi bien dans les 3 zones que dans la RBF prise globalement. Cette classe est suivie par celle des hauteurs comprises entre 0 et $2 \mathrm{~m}$ avec $23 \%$ des individus, puis par la classe de hauteur] 4-6 m] qui regroupe $19 \%$ des ligneux de la RBF. Les trois premières classes qui regroupent les individus dont la hauteur est comprise entre 0 et $6 \mathrm{~m}$ représentent prés de $90 \%$ des arbres inventoriés, ce qui fait dire que les formations végétales de la réserve de biosphère sont dominées par les savanes arbustives. Les arbres dont la hauteur est supérieure à $6 \mathrm{~m}$ ne représentent que $10 \%$ des individus inventoriés.

Dans les différentes zones de la RBF, l'examen de la structure des populations des trois espèces les plus abondantes dans les différentes classes de hauteur (figure 5) permet d'apporter quelques indications subsidiaires sur ce peuplement. 


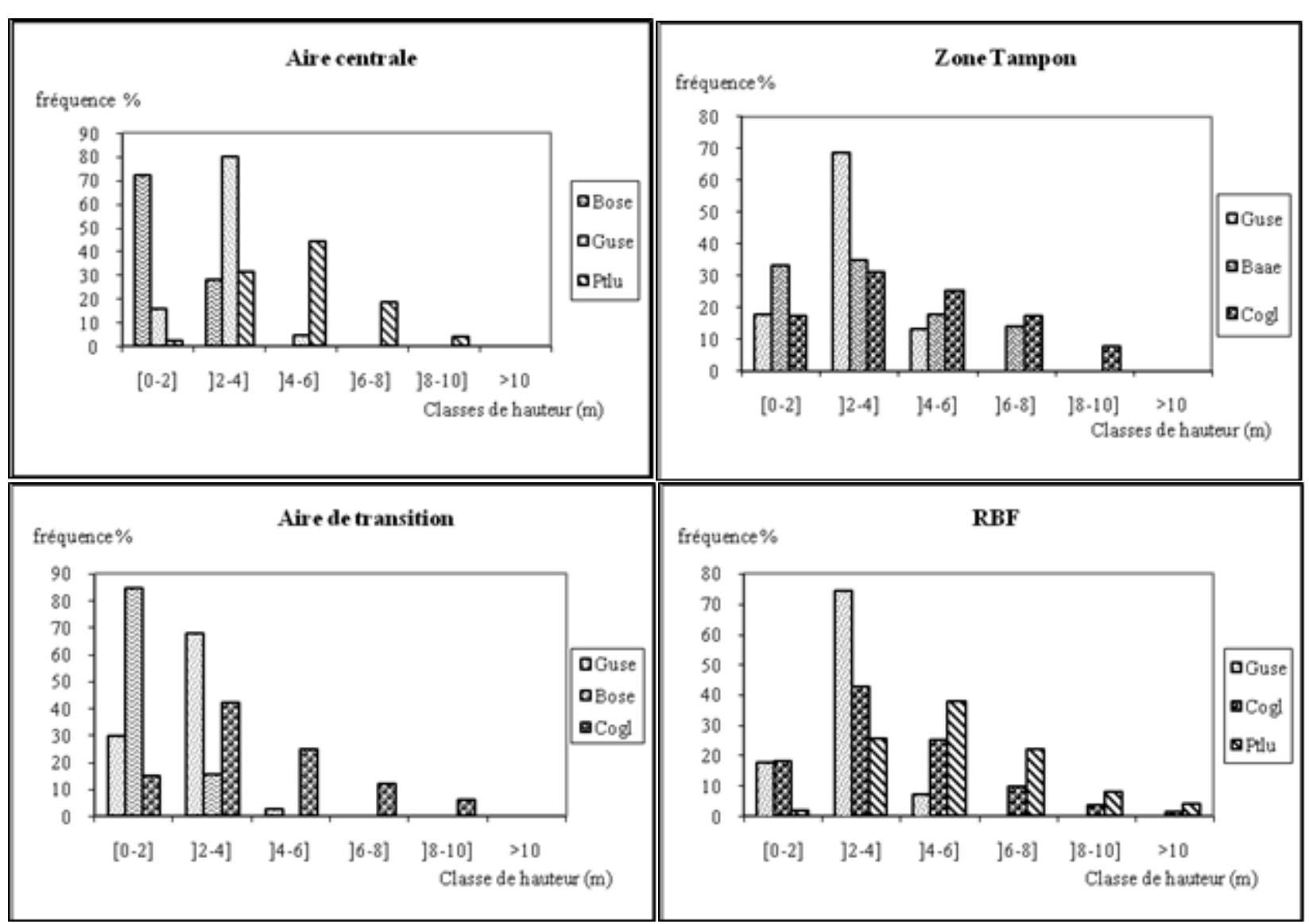

Figure 5 : Distribution des 3 espèces les plus abondantes selon les classes de hauteur dans la RBF (Guse $=$ Guiera senegalensis $;$ Bose $=$ Boscia senegalensis $;$ Ptlu $=$ Pterocarpus lucens $;$ Baae = Balanites aegyptiaca ; Cogl = Combretum glutinosum)

Dans l'aire centrale, la distribution de la population Boscia senegalensis a révélé que cette espèce n'est présente que dans les classes [0-2 m] et] 2-4 m] avec respectivement des fréquences de $72 \%$ et de $28 \%$. Pour Guiera senegalensis, la classe modale] 2-4 m] regroupe $80 \%$ des individus contre $15,6 \%$ pour la classe [0-2]. Ces deux espèces ne présentent aucun individu dont la hauteur est $>6 \mathrm{~m}$. L'absence de catégories supérieures pour ces espèces semble être liée au fait qu'elles sont des arbustes souvent coupés lors des défrichements, mais également pour produire du bois de feux et de services. La structure de Pterocarpus lucens diffère de celle des deux espèces précédentes du fait d'une présence remarquée dans les classes supérieures $; 67 \%$ des individus inventoriés ont une hauteur comprise entre 4 et $10 \mathrm{~m}$. En zone tampon, la structure des trois espèces les plus abondantes est de type unimodal et est comparable à la structure du peuplement de la RBF qui montre une prédominance des individus dans la classe]
2-4 m]. Cependant, les classes] 4-6 m] et] 6-8 m] sont représenté pour Balanites aegyptiaca et Combretum glutinosum. Dans l'aire de transition la structure des trois espèces les plus abondantes est caractérisée par une forte présence de Boscia senegalensis dans la classe [02] (85\%) et de Guiera senegalensis dans la classe] 2-4] avec une fréquence de distribution de $68 \%$. Ces deux espèces sont absentes dans les classes supérieures, contrairement à Combretum glutinosum qui présente une structure plus équilibrée.

* Distribution selon la circonférence : Dans la RBF, la circonférence des ligneux inventoriés est comprise entre $10 \mathrm{~cm}$ (Guiera senegalensis) et $808 \mathrm{~cm}$ (Adansonia digitata). La distribution des arbres selon les classes de circonférence (figure 6) montre que la structure des peuplements est similaire dans les différentes zones de la réserve de biosphère. Dans la classe $[10-50 \mathrm{~cm}]$, on retrouve $85 \%$ des individus dans l'aire centrale $; 72,8 \%$ des individus de la zone tampon et 
$76,2 \%$ des arbres dans l'aire de transition. Les autres représentées mais avec une structure comparables dans classes de circonférence sont ainsi faiblement les différentes zones de la réserve de biosphère.

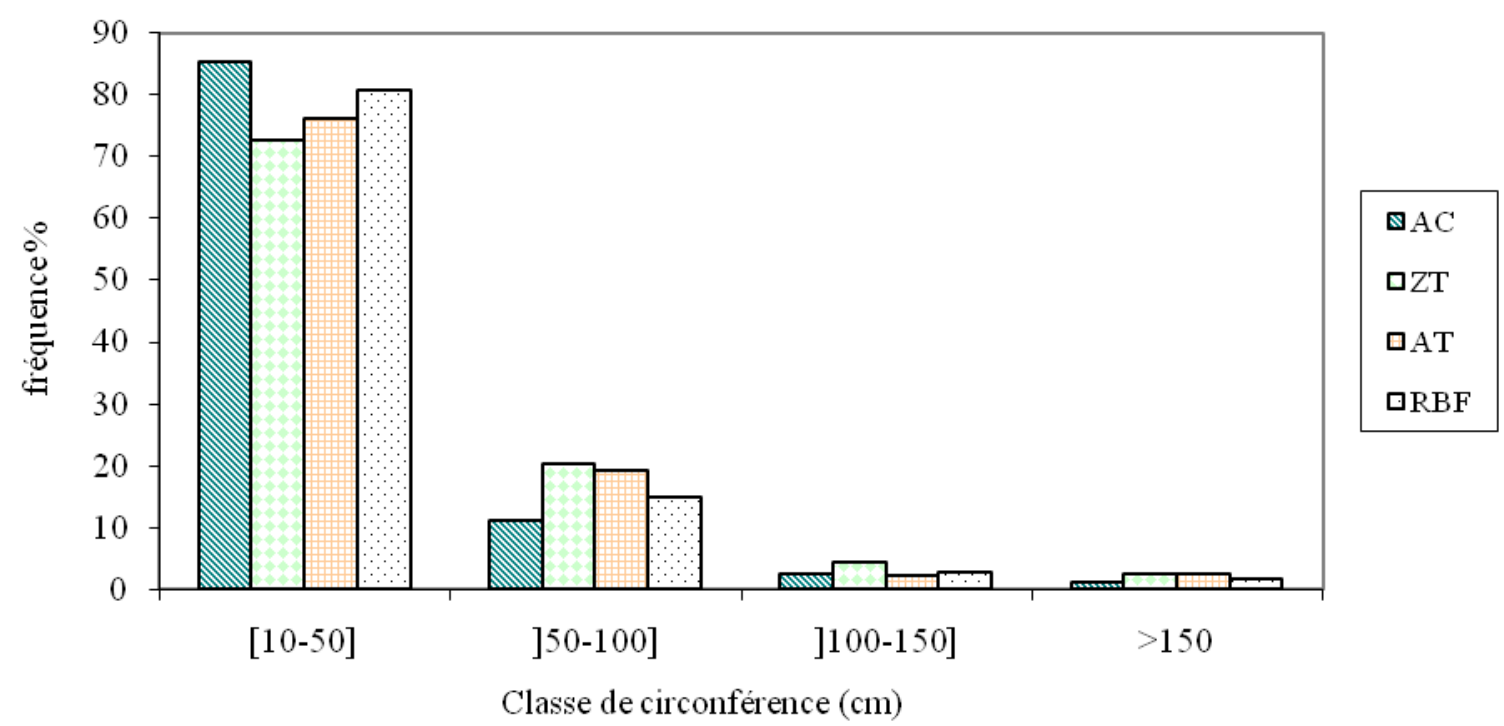

Figure 6 : Distribution des ligneux dans la RBF selon les classes de circonférence $(\mathrm{cm})$

Dans les différentes zones de la réserve de biosphère, nous avons établi les histogrammes de distribution des populations des trois espèces les plus abondantes en fonction des classes de circonférence (figure 7). L'examen de ces graphiques montre qu'ils présentent approximativement les mêmes allures. Ils sont caractérisés par la prédominance de la classe [10-50 cm]. En effet, dans l'aire centrale $98 \%$ des effectifs de Boscia senegalensis et $99 \%$ des individus de Guiera senegalensis se retrouvent dans cette classe. En zone tampon et dans l'aire de transition la fréquence de présence de Guiera senegalensis, Boscia senegalensis et Balanites aegyptiaca atteint des pics qui varient entre 80 et $95 \%$. Toutefois il faut noter la présence d'espèces à gros diamètre surtout en zone de transition et en zone tampon du fait de l'omniprésence de l'homme qui intervient dans la sélection de certaines espèces. Ces espèces à gros tronc sont principalement Sterculia setigera et Adansonia digitata. 

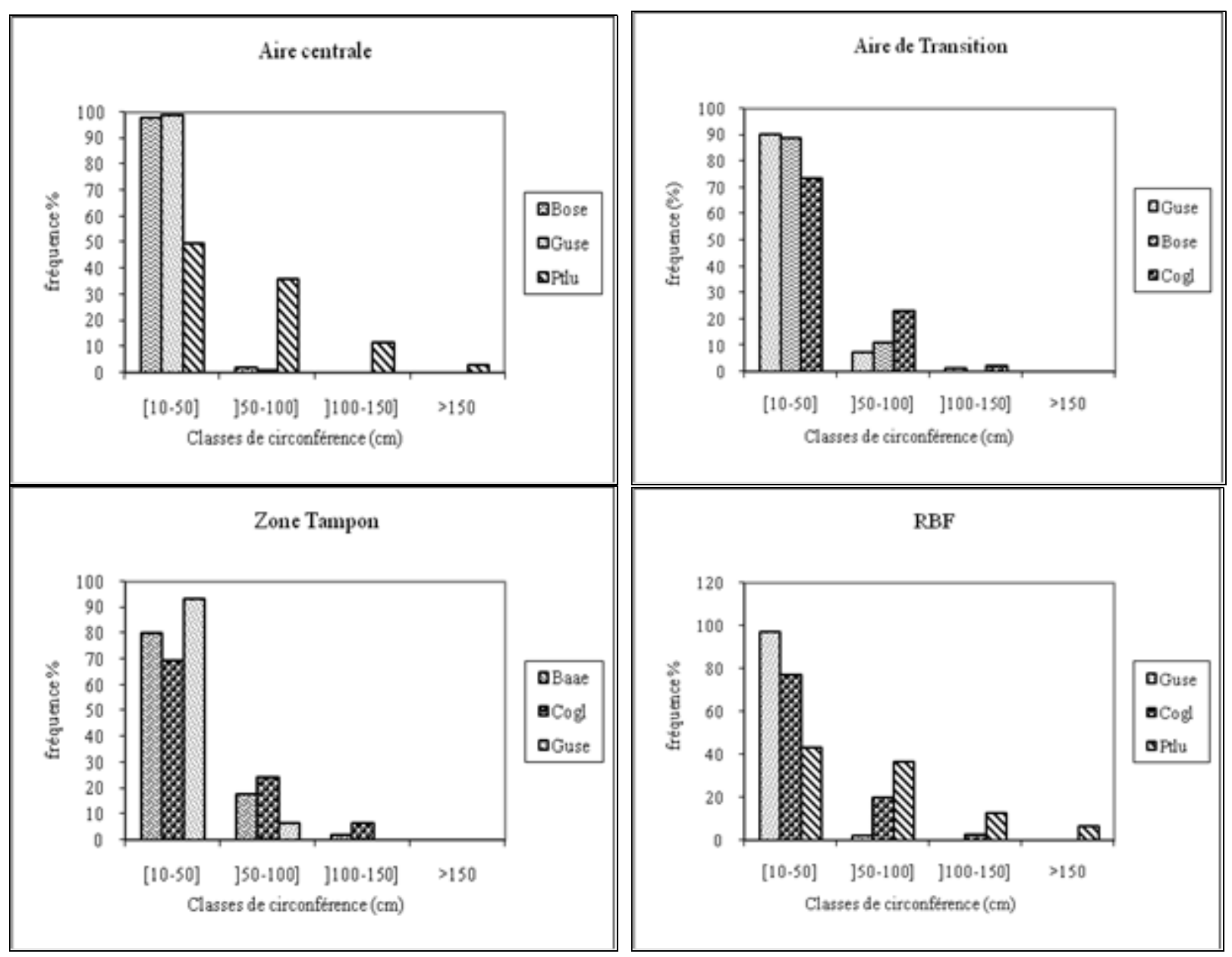

Figure 7 : Distribution des 3 espèces les plus abondantes, selon les classes de circonférence dans la RBF (Guse $=$ Guiera senegalensis $;$ Bose $=$ Boscia senegalensis $;$ Ptlu $=$ Pterocarpus lucens $;$ Baae $=$ Balanites aegyptiaca $;$ Cogl $=$ Combretum glutinosum)

La distribution du peuplement ligneux selon la hauteur et la grosseur dans la réserve de biosphère montre que la végétation est dominée par des arbustes; il s'agit de savanes arbustives.

Importance écologique : L'importance écologique des espèces (Importance Value Index) ou IVI de Curtis et Macintosh (1951) est calculée pour les différentes zones de la RBF. L'analyse du tableau 4 révèle que globalement les espèces prépondérantes, qui présentent des valeurs d'importance écologique les plus élevées sont Pterocarpus lucens, Guiera senegalensis et Combretum glutinosum avec respectivement des valeurs de $18,1 \% ; 16,09 \%$ et $13,4 \%$. Dans l'aire centrale, les espèces avec des valeurs d'IVI les plus élevées sont Pterocarpus lucens (22,9\%), Guiera senegalensis $(20,9 \%)$ et Boscia senegalensis $(10,56 \%)$. En zone tampon, les espèces les plus importantes sont les mêmes que dans l'ensemble de la RBF à savoir Pterocarpus lucens, Guiera senegalensis et Combretum glutinosum. Dans l'aire de transition Combretum glutinosum avec une IVI de 23,48\% est l'espèce prépondérante devant Guiera senegalensis $(9,02 \%)$ et Boscia senegalensis (7,84\%). Pterocarpus lucens qui a l'IVI la plus élevée dans l'aire centrale et la zone tampon est moins présente dans l'aire de transition caractérisée par une forte anthropisation. 
Tableau 4 : Valeurs d'importance écologique (\%) des espèces les plus représentatives dans les trois zones de la RBF

\begin{tabular}{|c|c|c|c|c|}
\hline Espèces $\quad$ Zone & Aire centrale & Zone tampon & Aire de transition & RBF \\
\hline Pterocarpus lucens & 22,93 & 22,51 & 5,34 & 18,10 \\
\hline Guiera senegalensis & 20,87 & 12,89 & 9,02 & 16,09 \\
\hline Combretum glutinosum & 8,28 & 12,68 & 23,48 & 13,40 \\
\hline Boscia senegalensis & 10,56 & 3,38 & 7,84 & 8,14 \\
\hline Grewia bicolor & 8,05 & 4,98 & 3,97 & 6,20 \\
\hline Adansonia digitata & 7,08 & 0,76 & 4,58 & 4,57 \\
\hline Balanites aegyptiaca & 1,86 & 8,93 & 5,83 & 4,56 \\
\hline
\end{tabular}

La régénération naturelle du peuplement: La régénération naturelle est à la base de la compréhension de la dynamique de la végétation ligneuse. Elle peut être végétative ou par semis naturel. Elle passe par le recrutement, la mortalité juvénile et les différents stades de développement, puis la survie (Traoré, 1997). Dans la réserve de biosphère, la régénération du peuplement a été évaluée par l'importance des jeunes plants (circonférence $<10 \mathrm{~cm}$ ). Le taux de régénération du peuplement est de $72 \%$ dans l'ensemble de la RBF. II varie fortement d'une zone à l'autre. II est deux fois plus élevé dans l'aire centrale $(79 \%)$ qui est moins anthropisée que dans la zone tampon (36\%) et l'aire de transition (39\%) (Tableau 3). L'importance de la régénération en fonction des différentes espèces a été appréhendée par le calcul de l'indice spécifique de régénération (ISR) dans les 3 zones de la RBF (tableau $5)$.

Tableau 5 : Indice spécifique de régénération (ISR) en \% dans les trois zones de la RBF

\begin{tabular}{|c|c|c|c|c|}
\hline \multicolumn{1}{|c|}{ Zone } & Aire centrale & Zone tampon & Aire de transition & RBF \\
\hline Guiera senegalensis & 64,43 & 52,41 & 38,34 & 62,37 \\
\hline Boscia senegalensis & 17,16 & 11,40 & 8,29 & 16,38 \\
\hline Combretum glutinosum & 3,86 & 12,06 & 14,51 & 4,86 \\
\hline Grewia bicolor & 4,36 & 4,82 & 1,73 & 4,23 \\
\hline Balanites aegyptiaca & 1,85 & 11,62 & 15,03 & 3,05 \\
\hline Pterocarpus lucens & 3,00 & 0,88 & 1,73 & 2,83 \\
\hline Combretum micranthum & 2,01 & 0,44 & 0,17 & 1,83 \\
\hline
\end{tabular}

L'examen du tableau 5 montre que Guiera senegalensis a le meilleur potentiel de régénération de la RBF avec un indice spécifique de régénération de $62,37 \%$. Elle est suivie de Boscia senegalensis avec 16,38\%. Ces deux espèces comportent $78 \%$ des jeunes plants inventoriés dans la RBF.Dans l'aire centrale Guiera senegalensis $(64,43 \%)$ et Boscia senegalensis $(17,16 \%)$ ont le plus contribué au potentiel de régénération du peuplement ligneux. En fait, plus de $80 \%$ des plants juvéniles inventoriés dans cette zone relèvent de ces deux

\section{DISCUSSION ET CONCLUSION}

L'objectif de ce travail est de caractériser le peuplement végétal de la RBF. Nous avons alors examiné espèces. En zone tampon Guiera senegalensis (52,41\%) s'accompagne de Combretum glutinosum $(12,06 \%)$ et Balanites aegyptiaca $(11,62 \%)$. Ces trois espèces présentent également les potentiels de régénération les plus élevés dans l'aire de transition avec des proportions différentes. Globalement, les espèces qui présentent les indices spécifiques de régénération les plus élevés sont les arbustes notamment les Combretaceae qui régénèrent facilement par rejets de souche en l'absence des feux de brousse.

successivement le cortège floristique, la distribution spatiale du peuplement, l'importance écologique et le 
potentiel de régénération du peuplement. Dans la RBF, la flore est riche de 49 espèces réparties en 32 genres relevant de 16 familles botaniques. II importe de noter que la fiabilité de la richesse spécifique dépend de l'exhaustivité de l'inventaire. Or les relevés ne sont jamais exhaustifs car il y a un problème de détectabilité des espèces (Gosselin \& Laroussinie, 2004 ; Deconchat \& Balent, 2004). II faut cependant noter que la richesse spécifique n'est qu'un des indicateurs, des descripteurs de la biodiversité. L'analyse des fréquences centésimales a montré que Guiera senegalensis est l'espèce la plus fréquente dans la réserve avec une présence dans les $3 / 4$ des relevés effectués. Elle est suivie de Combretum glutinosum $(65,5 \%)$, Boscia senegalensis $(63,6 \%)$ et Pterocarpus lucens $(60,9 \%)$. Ces résultats révèlent que les Combretaceae (Guiera senegalensis et Combretum glutinosum) sont très fréquentes au Ferlo et occupent de plus en plus d'espaces, ce qui fait dire à Ngom (2008) que ces deux espèces sont entrain de coloniser le milieu avec comme corollaire une combrétinisation et une modification de la structure de la végétation ligneuse. $\mathrm{Ce}$ sont des espèces caractéristiques, de bons indicateurs des changements d'état de la végétation ligneuse. La densité réelle du peuplement qui est de 389 pieds/hectare dans l'ensemble de la réserve varie en fonction des différentes zones. Elle est plus élevée dans l'aire centrale (392) que dans la zone tampon (352) et l'aire de transition (347). Cet état de fait s'explique par la plus faible présence de l'homme dans l'aire centrale que dans les autres unités. Le rapport entre la densité théorique et la densité observée $(2,22)$ est très élevée dans la RBF ; ce qui traduit une distribution en agrégats de la végétation; avec la présence tantôt d'endroits très clairsemés, tantôt d'endroits où les individus sont en bosquets (Gning, 2008). L'aire centrale qui a la densité observée la plus élevée de la réserve de biosphère, présente la surface terrière la plus faible parce que la flore est dominée par Guiera senegalensis et Boscia senegalensis qui sont des arbustes, avec des troncs de faible grosseur. Ceci confirme Bouxin (1975) selon qui il n'existe pas de parallélisme entre la surface terrière et la densité.

Le taux de recouvrement est de $35,3 \%$ dans l'ensemble de la RBF. Ce couvert végétal plus élevé en zone tampon $(43 \%)$ que dans les autres unités s'explique par la présence d'arbres à grandes cimes (Adansonia digitata, Sterculia setigera, Pterocarpus lucens). En effet, Les arbres à grands houppiers contribuent plus au recouvrement et jusqu'à un certain degré de recouvrement, ils modifient les conditions écologiques en réduisant le pouvoir évaporant de l'air, en favorisant le bilan hydrique du sol et en améliorant la fertilité (Akpo, 1993). La structure du peuplement ligneux de la RBF établie suivant les classes de hauteur montre que les trois premières classes qui regroupent les individus dont la hauteur est comprise entre 0 et $6 \mathrm{~m}$ représentent prés de $90 \%$ des arbres inventoriés. Ceci révèle l'importance de la strate arbustive dans les 3 zones constitutives de la réserve de biosphère. La structure du peuplement selon les classes de circonférence montre que $95,7 \%$ des individus inventoriés ont des circonférences comprises entre 0 et $1 \mathrm{~m}$. Ceci confirme la forte proportion d'individus relativement jeunes, constituant une savane arbustive. L'importance des individus jeunes du peuplement provient en fait essentiellement des populations de Guiera senegalensis, Boscia senegalensis et dans une moindre mesure de Combretum glutinosum et Pterocarpus lucens. D'une manière générale, la variation des communautés végétales dans la RBF parait être liée plus à l'anthropisation (surpâturage, feux de brousse, coupe des arbres, défrichement...) qu'aux conditions intrinsèques des systèmes d'utilisation des terres (nature des sols, disponibilité en eau....). Les variations de la disponibilité en eau des sols constituent toutefois une des causes principales de l'hétérogénéité spatiale des communautés végétales. Ces variations peuvent dépendre aussi de l'hétérogénéité de la couverture pédologique qui conditionne la redistribution de l'eau à l'échelle des versants sous l'action du ruissellement (Olswig-Whittaker et al, 1983). La structure spatiale de la végétation intervient elle-même dans la redistribution de l'eau; sa dynamique naturelle ou provoquée engendre soit un renforcement des processus existants, soit une évolution vers des modes de fonctionnement nouveaux (Cornet, 1992). Globalement, dans la réserve de biosphère, trois espèces se dégagent de par leur importance écologique : Pterocarpus lucens, Guiera senegalensis et Combretum glutinosum. Les deux derniers à savoir les Combretaceae (Combretum glutinosum et Guiera senegalensis) ont marqué par leur présence l'écosystème. Ces deux espèces sont entrain de coloniser le milieu avec comme corollaire une combrétinisation et une modification de la structure de la végétation ligneuse. Pterocarpus lucens qui est surtout présente dans l'aire centrale et en zone tampon joue un rôle important dans l'apport fourrager en saison sèche. En effet, Pterocarpus lucens est une des espèces les plus appétées et les plus nutritionnelles des espèces fourragères ligneuses sahéliennes (Wilson, 1980). Le taux de régénération du peuplement végétal est de $72 \%$ 
dans l'ensemble de la RBF. II est deux fois plus élevé dans l'aire centrale $(79 \%)$ que dans la zone tampon $(36 \%)$ et l'aire de transition (39\%). Ceci est lié au fait que l'aire centrale qui bénéficie d'un statut légal de protection subit moins la pression de l'homme que les deux autres zones où des établissements humains sont installés et où tous les systèmes d'utilisation des terres sont rencontrés. L'importance de cette régénération varie selon les différentes espèces. Guiera senegalensis est la seule espèce qui présente un potentiel de régénération acceptable avec un indice spécifique de régénération de $62 \%$. En effet, cette espèce est capable de régénérer même après une coupe rase par apparition de rejets de souches appétées par les bovins. Elle est suivie de Boscia senegalensis qui regroupe $16 \%$ de l'ensemble des jeunes individus inventoriés. Balanites aegyptiaca a également un potentiel de régénération moyen dans la zone de transition ou zone de coopération. Elle colonise tous les sols et toutes les situations topographiques. Sa régénération semble se faire par semis naturel contrairement à Guiera senegalensis. Ce semis naturel est facilité par la consommation des fruits par les animaux qui rejettent les amandes dans les déjections, ce qui facilite la germination (Ngom, 2008). Selon Grouzis et Albergel (1988), cité par Gning (2008), en zone

\section{REFERENCES BIBLIOGRAPHIQUES}

Adou Yao CY, et Nguessan EK, 2005. Diversité taxonomique dans le sud du parc national de Taï, Côte d'Ivoire. Rev. Afrique Science (01) (2) (2005) : 295-313.

Akpo LE, 1993. Influence du couvert ligneux sur la structure et le fonctionnement de la strate herbacée en milieu sahélien. Orstom éd., TDM, $174 \mathrm{p}$.

Akpo LE, 1998. Effets de l'arbre sur la végétation herbacée dans quelques phytocénoses du Sénégal : Variation selon un gradient climatique. Thèse de Doctorat d'Etat en Sciences Naturelles, UCAD : $142 \mathrm{p}$.

Akpo LE et Grouzis M, 1996. Influence du couvert sur la régénération de quelques espèces ligneuses sahéliennes (Nord-Sénégal, Afrique occidentale). Webbia 50 (2) : 247-263.

Arbonnier M, 2002. Arbres, arbustes et lianes des zones sèches d'Afrique de l'Ouest. CIRAD et Museum d'histoire naturel de Paris : $573 \mathrm{p}$.

Berhaut J, 1967. Flore du Sénégal. Edition clair Afrique

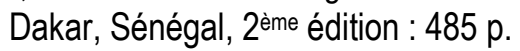

sahélienne, les capacités de régénération résident dans les caractères d'adaptation des espèces et des structures de végétation face à la sècheresse et à la variabilité des conditions édapho-climatiques. Aussi, il importe de noter que le passage du feu stimule le rejet de souches chez certaines espèces notamment Guiera senegalensis et Boscia senegalensis. En effet, les perturbations qui détériorent la partie aérienne de l'écosystème, mais qui préservent le sol, favorisent les espèces qui drageonnent, qui rejettent des souches ou qui régénèrent à partir de la banque de semences du sol (Gosselin \& Laroussinie, 2004). En dehors de ces trois espèces, le potentiel de régénération est globalement faible pour les autres espèces présentes dans la RBF. Le surpâturage, la péjoration climatique, les feux de brousse et la surexploitation semblent en être les principales causes. Globalement, la RBF présente des potentialités intéressantes en matière de ressources végétales si on tient compte de la densité arborée, du couvert végétal, de la structure de la végétation et de la capacité de régénération. Ces résultats montrent que la RBF est capable d'assurer le double rôle de conservation de la biodiversité et de fourniture de services écosystèmiques aux communautés locales en particulier.

Boudet G, 1984. Manuel sur les pâturages tropicaux et les cultures fourragères. 4ème édition. Paris, Ministère de la Coopération, Manuel et Précis d'élevage 4, 254p.

Bouxin G, 1975. Ordination and classification in the savana vegetation of the Akagera Park (Rwanda, Central Africa). Vegetation 29 : 155157.

Cornet A, 1992. Relation entre la structure spatiale des peuplements végétaux et le bilan hydrique des sols de quelques phytocénoses en zone aride. In L'aridité une contrainte au développement. ORSTOM, Editions, 1992. 245-263.

Gning ON, 2008. Caractéristiques des ligneux fourragers dans les parcours communautaires de Khossanto (Kédougou, Sénégal Oriental). Mémoire de DEA en Biologie végétale, UCAD, $78 p$.

Gounot M, 1969. Méthodes d'études quantitatives de la végétation. Paris, Masson et Cie.

Gouzis M et Albergel J, 1991. Du risque climatique, à la contrainte écologique: incidences de la sécheresse sur les productions végétales et le 
milieu au Burkina Faso. in "Le risque en Agriculture », ELDIN M. et MILLEVILLE P., Ed., Collection à traves champs, ORSTOM, Paris : 243-254

Kouame NF, 1998. Influence de l'exploitation forestière sur la végétation et la flore de la forêt classée du Haut-Sassandra (Centre-Ouest de la Côte d'lvoire). Thèse de $3^{\text {ème }}$ cycle univ. de Cocody (Côte d'Ivoire), 227p.

Labat JN, 1995. Végétation du nord-ouest du Michoacan Mexique. Instituto de Ecologia A. C., 401p.

Le Houerou HN, 1989. The grazing land ecosystems of the African Sahel. Springer-verlag, Berlin : 282p-

Long G, 1974. Diagnostic phyto-écologique et aménagement du territoire. I. Principes généraux et méthodes. Masson, Paris : $252 \mathrm{p}$.

Mori SA, Boom BM, De Carvalino AM, et Dos Santos TS, 1983. Southern Bahia moist forest. Bot. Rev. 49(2) (1983) pp. 155-232.

Ngom D, 2008. Définition d'indicateurs de gestion durable des ressources sylvo-pastorales au Ferlo (Nord-Sénégal), Thèse de Doctorat en Biologie végétale, Option: Écologie, FST, UCAD, 148p.

Olswig-Whitaker L, Shachak M et Yair A. 1983. Vegetation patterns related to environmental factors in a Negev Desert Watershide. Vegetatio, 54 : 153-165.

Pohle GW et Thomas MLH, 2001. Protocole de surveillance du benthos marin: Macrofaune intertidale et infratidale. Le réseau d'évaluation et de surveillance écologiques, Canada. Site : http://www.eman-

rese.ca/rese/ecotools/protocols/marine/benthos/ benthos4.html

Poupon $\mathrm{H}, 1980$. Structure et dynamique de la strate ligneuse d'une steppe sahélienne au nord du Sénégal. ORSTOM éd. (Etudes \& Thèses), Paris : $307 p$.

Ramade F, 2003. Eléments d'Écologie : Écologie

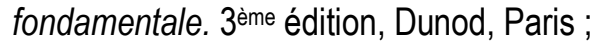
$690 \mathrm{p}$.

Toupet C, 1989. Comparaison des sécheresses historiques et de la sécheresse actuelle : essai de définition de la sécheresse et de l'aridification. In: BRET coord, Les hommes face aux sécheresses, Nord est brésilien, sahel africain : 77-84, EST-IHEAL éd. (422pp.).

Traoré SA, 1997. Analyse de la flore et de la végétation de la zone de Simenti (Parc National du Niokolo Koba), Sénégal oriental. Thèse de $3^{\text {ème }}$ cycle. FST/UCAD (Sénégal) : $136 \mathrm{p}$.

UNESCO, 1996. Réserves de biosphère : la Stratégie de Séville et le cadre statutaire du réseau mondial. UNESCO, Paris. 23p.

Wilson RT, 1980. Consommation de bois de combustion dans une ville du Mali central et ses effets sur la disponibilité des fourrages ligneux. In les fourrages ligneux en Afrique: Etat actuel des connaissances : 463-466. 